\section{Knowledge, attitude and practice on maternal immunization with tetanus toxoid, reduced diphtheria toxoid, acellular pertussis (Tdap) among pregnant women in Korea}

Taek-Jin Lee, Shin-Hye Lee, Bo-Kyeung Jin, Kyeung-Suk Baek, Yeung-Sun Cho

Department of Pediatrics, CHA Bundang Medical Center, CHA University, Seongnam, Korea

\section{Purpose}

In South Korea, the number of cases of pertussis reported has increased in recent years. Despite effectiveness of maternal tetanus toxoid, reduced diphtheria toxoid, acellular pertussis (Tdap) vaccination, immunization rates of Tdap during pregnancy remain quite low. We assessed the knowledge, attitude and practice on maternal Tdap vaccination among pregnant women.

\section{Methods}

This study was a cross-sectional survey of pregnant women who visited obstetrics and gynecologic units in the Gyeonggi-do province of Korea Individual questionnaires were administered to assess knowledge, attitude and practice on maternal immunization with Tdap.

\section{Results}

The questionnaires was completed by 184 pregnant women; 158 (86\%) had not received information from doctors about pertussis and Tdap, and $166(90 \%)$ did not know the need for Tdap vaccination. Only $7 \%$ of pregnant women unlikely to receive Tdap vaccine during current pregnancy answered 3 or more of the 5 knowledge-based questions correctly (Table 1, Fig. 1). By logistic regression analysis, recommendation by doctor (OR 236.2), belief that the vaccine is effective (OR 40.21), and belief that the vaccine is safe (OR 19.83) were significantly important factors to respondents' intention to be vaccinated (Table 2).

\section{Conclusions}

Most pregnant women seem to be neither recommended nor adequately informed about Tdap vaccination. Information given by health care professionals is very important to increase Tdap coverage among pregnant women.

Key Words: pertussis, immunization, pregnancy, attitude
Table 1. Maternal Characteristics and Associations with Intention to Receive Antenatal Tdap Vaccines

\begin{tabular}{|l|c|c|l} 
Characteristics & $\begin{array}{c}\text { Likely to receive } \\
\text { Tdap vaccine } \\
\text { during current } \\
\text { pregnancy }(n=18)\end{array}$ & $\begin{array}{c}\text { Unlikely to receive } \\
\text { dop vaccine } \\
\text { during current } \\
\text { pregnancy }(n=166)\end{array}$ & P value \\
\hline
\end{tabular}

Age (years), mean

$33 \quad 32 \quad 0.734$

Gestational age(weeks), mean $\quad 27 \quad 28 \quad 0.451$

Primiparous (\%)

0.026

Knowledge score $\geq 60 \%(\%)^{*}$

28

* Proportion of respondents who answered 3 or more of the 5 knowledge-based questions correctly. Tdap, tetanus toxoid, reduced diphtheria toxoid, acellular pertussis.

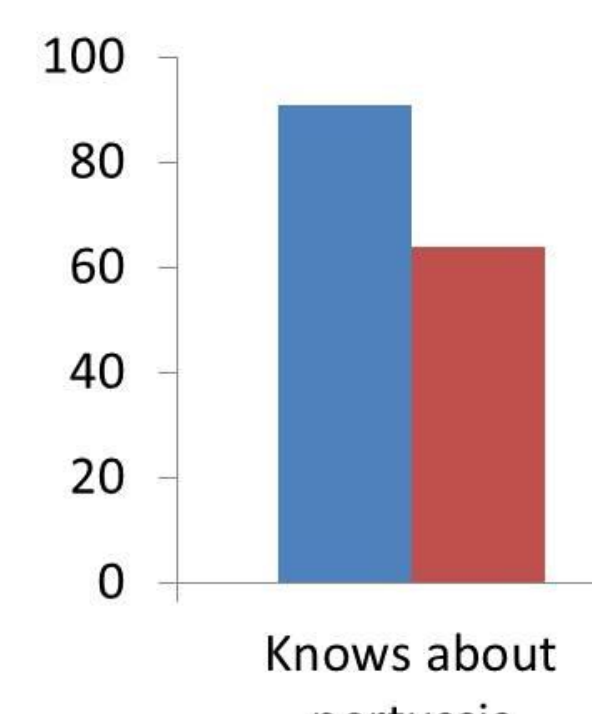

pertussis

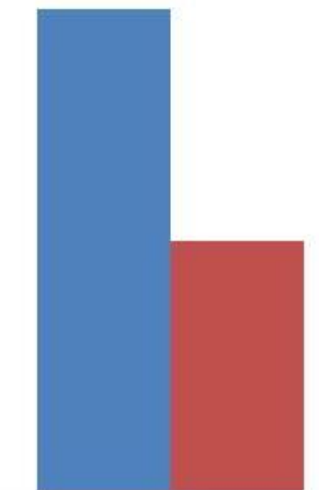

Knows about benefits of vaccines in preventing pertussis

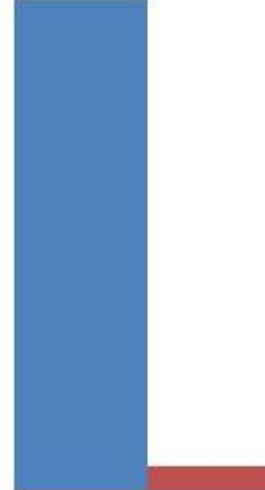

Knows Tdap vaccine Knows Tdap vaccine is recommended is safe for pregnant during pregnancy women and their babies
- Likely to receive Tdap vaccine during current pregnancy

- Unlikely to receive Tdap vaccine during current pregnancy

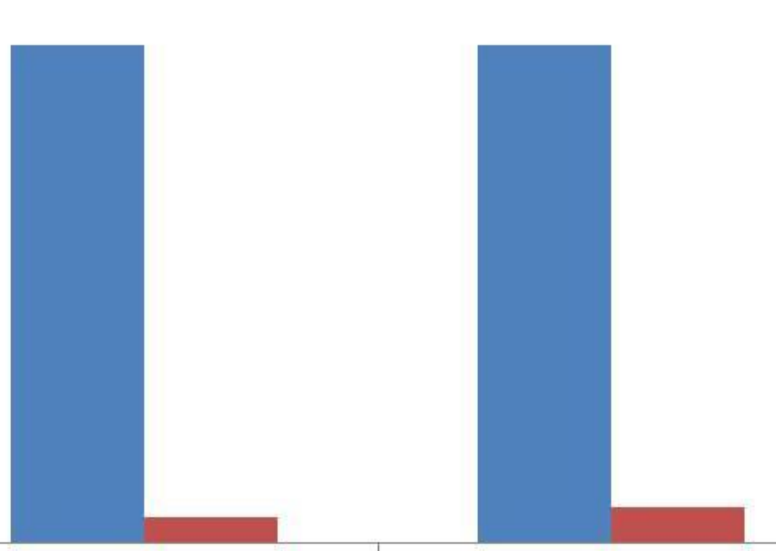

Knows after receiving Tdap vaccine, pro antibodies will be created and provide their babies shortterm protection against pertussis
Fig. 1. Comparison of proportion of respondents answered correctly among pregnant women who intended to receive Tdap (tetanus toxoid, reduced diphtheria toxoid, acellular pertussis) vaccine during pregnancy versus those who did not intend to receive it.

Table 2. Factors Associated with Intention to Receive Antenatal Tdap Vaccines Tdap, tetanus toxoid, reduced diphtheria toxoid, acellular pertussis.

\begin{tabular}{l|c|c|c|}
\hline Variables & OR & $95 \%$ CI & P value \\
\hline Recommendation by doctor & 236.2 & $12.6,4431.9$ & $<0.001$ \\
\hline Belief that the vaccine is effective & 40.21 & $2.35,687.7$ & 0.011 \\
\hline Belief that the vaccine is safe & 19.83 & $1.54,255.9$ & 0.022 \\
\hline Cost of vaccine & 1.27 & $0.002,683.9$ & 0.942 \\
\hline
\end{tabular}

Tdap, tetanus toxoid, reduced diphtheria toxoid, acellular pertussis. 